\title{
Automated Planning of Computer Assisted Mosaic Arthroplasty
}

\author{
Jiro Inoue ${ }^{1,3}$, Manuela Kunz ${ }^{1,3}$, Mark B. Hurtig ${ }^{4}$, \\ Stephen D. Waldman ${ }^{2,3}$, and A. James Stewart ${ }^{1,3}$ \\ 1 School of Computing \\ 2 Department of Mechanical Engineering, Queen's University \\ ${ }^{3}$ Human Mobility Research Centre, Kingston General Hospital \\ ${ }^{4}$ Ontario Veterinary College, University of Guelph
}

\begin{abstract}
We describe and evaluate a computer algorithm that automatically develops a surgical plan for computer assisted mosaic arthroplasty, a technically demanding procedure in which a set of osteochondral plugs are transplanted from a non-load-bearing area of the joint to the site of a cartilage defect. We found that the algorithm produced plans that were at least as good as a human expert, had less variability, and took less time.
\end{abstract}

\section{Introduction}

Cartilage degeneration is a widespread problem which occurs predominantly in the knee, ankle, and shoulder. Articular cartilage is not vascularized and does not naturally repair when damaged [1]. In the event of traumatic injury, this can lead to long-term damage and a loss of bearing capacity in the joint surface.

Mosaic arthroplasty is an operation that repairs damaged cartilage by transplanting osteochondral plugs from a non-load-bearing part of the joint to the site of the cartilage defect 23 .

Mosaic arthroplasty is a technically challenging operation. The osteochondral plugs must be delivered so as to exactly reconstruct the original surface. But the top surface of a plug is curved and may not be perpendicular to the axis of the plug, so the position and orientation of each plug must be planned and achieved.

Plugs that are too high can result in poor plug integration due to micromotion and increased contact pressure 415. Plugs that are too low can result in cartilage necrosis and fibrocartilage overgrowth [6]. A plug with an angled surface that is incorrectly delivered may be too high in some parts and too low in other parts. The percentage of the repair surface that is too high is inversely correlated with the quality of healing [7].

Computer assisted mosaic arthroplasty (CAMA) uses computer planning and intraoperative guidance to overcome these problems. The surgical plan indicates the position and orientation of each plug's harvest location and delivery location. The surgeon follows the plan using optically tracked surgical instruments or patient-specific instrument guides 8 .

G. Fichtinger, A. Martel, and T. Peters (Eds.): MICCAI 2011, Part I, LNCS 6891, pp. 267-274, 2011.

(C) Springer-Verlag Berlin Heidelberg 2011 
The surgical plan is critical to the success of the operation. In the surgical plan:

- the original articular surface must be predicted as a reconstruction goal;

- the reconstructed cartilage thickness must match the original cartilage thickness to avoid degeneration at the bone/cartilage interface;

- the plug harvest sites must be planned so that surface orientation of each plug matches the predicted surface orientation at the corresponding delivery site; and

- an optimal pattern of plugs must be planned to cover the defect site with minimal overlapping and minimal gapping.

Manual planning for CAMA has been shown to be effective at finding harvest and delivery sites to reconstruct the original articular surface 9]. But manual planning is slow and requires a highly skilled human operator with knowledge of mosaic arthroplasty and the ability to use a 3D computer interface. The quality of a manually developed plan depends upon the skill of the operator and will be subject to inter-operator variability.

We describe a computer algorithm that automatically builds a surgical plan for CAMA. We show that the automatic planner achieves results that are at least as good as those of a skilled human operator and can reconstruct the correct surface to an accuracy of $0.3 \mathrm{~mm}$ RMS.

\section{Related Work}

Clinical evaluations have shown that surface congruency is critical to achieving a good repair. In an animal study 4, plugs that were too high were found to subside under weight bearing but showed fissuring, bone cysts, and poor bony incorporation. Elevated angled grafts (with an angled tip of the plug surface above the surrounding surface) are subject to increased contact pressure [5] which leads to peak loading and abrasion of the plug's surface [10]. On the other hand, plugs that are too low may be disposed to late degradation from inappropriate pressure [10] and have shown cartilage necrosis and fibrocartilage overgrowth [6].

Computer navigation of surgical tools has been shown to achieve greater plug perpendicularity and more appropriate plug depth [11] than with a freehand operation.

Computer planning and navigation have successfully been used in retrograde plug delivery in the human talus [12. The planning consisted of using patient images to manually choose a path for the surgical drill. Another study using the talus found better plug harvesting and placement with computer assist [13]. Computer planning has also been used to match plugs from the femoral condyles to defects in the talus [14.

Computer imaging has been used to match cartilage topography at harvest and defect sites. One study determined the congruity between the surfaces at a harvest site and a donor site by finding the rigid transformation between 


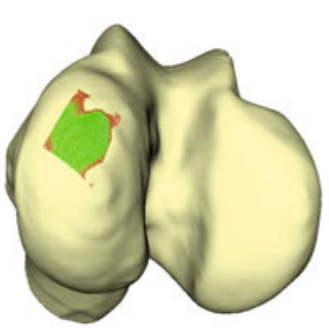

(a)

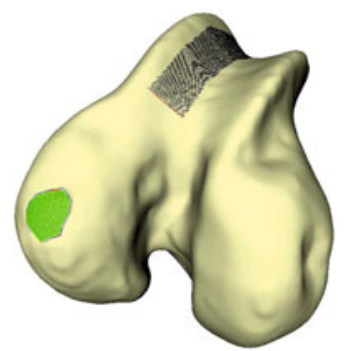

(b)

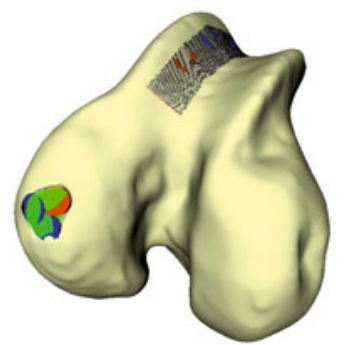

(c)

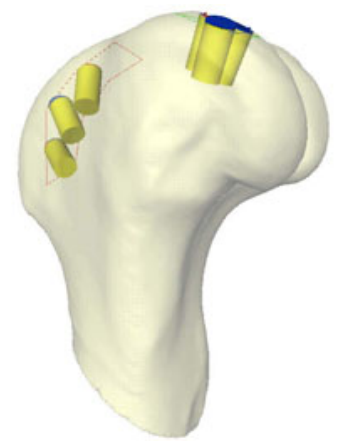

(d)

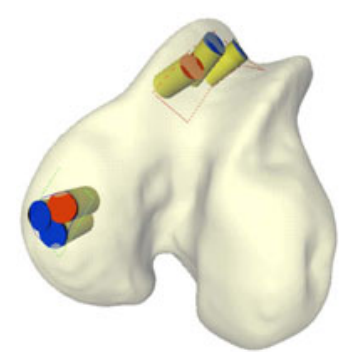

(e)

Fig. 1. The automatic planning process. (a) A bicubic spline is placed over the defect. (b) The defect area and harvest areas are outlined. (c) A pattern of plugs is made over the defect, and corresponding harvest sites found. (d,e) The final plan.

the surfaces that minimized the RMS error. The study applied this measure to seven cadaveric knees to propose the best harvest sites for particular defect sites [15. Another study on six dog femurs used similar topographic matching and considered different plug diameters, finding that larger plug diameters had fewer good harvest sites 16.

Computer planning requires that the original articular surface be estimated, since it is no longer present at the site of the defect. A cubic spline can be used to accurately predict the original articular surface [17.

\section{The Planning Algorithm}

We found that a surprising simple planning algorithm could obtain results at least as good as those of a human operator.

The input to the algorithm consists of two coregistered triangle meshes: one of the cartilage surface and one of the underlying bone. These meshes can be obtained from segmented MRI or CT-arthrogram images. The output of the algorithm consists of the harvest and implant positions and orientations of a set of plugs, along with the plug dimensions. 
In a preprocessing step, the original articular surface is estimated with a bicubic spline: The operator uses a 3D interface to pick four points on the cartilage surface around the defect and the algorithm creates a bicubic spline over the defect that interpolates those points and has the same tangent plan at those points (Figure 1(a)). This method has been shown to produce a surface within $0.27 \mathrm{~mm}$ RMS of the original, pre-defect surface [17.

Next, the operator outlines the defect on the spline surface. This is not done automatically because the defective cartilage usually extends beyond the area of visibly depressed cartilage (Figure 1(b)).

Next, the operator outlines areas of the joint from which cartilage plugs may be harvested (Figure 1(b)). This could be done automatically (but currently is not) by incorporating known good donor sites [1516.

Finally, the algorithm is executed. The first phase of the algorithm builds an optimal pattern of plugs at the defect site. The second phase finds optimal harvest locations for each of the plugs. Results are shown in Figure 1(c,d,e).

\section{Phase 1: Build plugs over defect}

A hexagon grid is placed on the spline surface over the defect area. The initial position and orientation of the grid are arbitrary. Hexagon centers are spaced $\sqrt{3} r$ apart for plugs of radius $r$ (in our case, $r=2.25 \mathrm{~mm}$ ). A plug is placed at each hexagon center, oriented perpendicular to the spline surface.

The pattern of plugs should cover as much of the defect as possible while minimizing the overlapping of plugs, since plug stability may be reduced by overlapping [18. To achieve this, the algorithm finds a pattern of plugs to minimize the cost function

$$
A_{\text {uncovered }}+0.4 \sum_{i} A_{i}
$$

where $A_{\text {uncovered }}$ is the area of the defect not covered by any plug and $A_{i}$ is the area of the $i^{\text {th }}$ plug. An area covered by multiple plugs is counted multiple times in the sum, which discourages overlapping, but not at the expense of too much uncovered area. The constant 0.4 was chosen through experimentation to balance the number of plugs against the uncovered area.

For a given pattern of plugs, the cost function is approximated by sampling the defect area on a $0.1 \mathrm{~mm}$ square grid. Each grid point that is not covered by a plug counts $(0.1 \mathrm{~mm})^{2}$ and each plug of radius $r$ counts $0.4 \pi r^{2}$.

Ten thousand iterations of simulated annealing are performed to minimize the cost function. In each iteration, three cases are considered: add a random plug; remove a random plug; and keep the same plugs. In each of the three cases, the position of every plug is perturbed uniformly randomly within a $k r \times k r$ square centered at the plug's current position on the spline surface, where $k$ is the "annealing temperature" which starts at one and decreases linearly with each iteration until it becomes zero in the last iteration. Of the three patterns considered, only the pattern of minimum cost is carried to the next iteration.

Note that plugs over the defect site are placed perpendicular to the surface. The algorithm could be extended to permit slanted plugs and to penalize subsurface plug intersection, in which a plug may be undercut by another plug, 
making the undercut plug more likely to loosen. We also consider plugs of only one radius, although it would be easy for the algorithm to choose random radii from the set of radii available among the surgical tools.

\section{Phase 2: Find harvest locations}

Given a pattern of plugs over the defect, the second phase finds optimal locations at which to harvest those plugs. This is done in a greedy fashion: The plugs are randomly ordered and an optimal harvest location is found for each plug, in order.

Since the order affects the harvest locations (i.e. plugs that are later in the order have fewer locations from which to choose) the algorithm picks ten random orderings of the plugs, tries each, and chooses the ordering that results in the best harvest locations.

To find the best harvest location for a particular plug, a $0.3 \mathrm{~mm}$ square grid is placed over the harvest areas and each grid point is considered as a harvest location. (Grid points that are within one diameter of an already-harvested plug are discarded because the joint is weakened when harvest holes are too close together.) For a particular grid point, the plug from the defect site is translated so that the middle of its top surface is coincident with the grid point and 49 orientations are considered by varying the angle from perpendicular in 5 degree increments from -15 to +15 degrees in both the $x$ and $y$ directions of the grid. At each orientation, the rotation around the plug axis and the translation along the plug axis are determined that give the best fit between the surface of the plug and the surface at the harvest site. The best fitting plug is chosen and its RMS surface error is used as the cost.

A variant of the algorithm tries to match both the cartilage surface and the bone surface. In this case, 49 orientations are again considered and the best axial rotation and translation are found for the cartilage surface and, separately, for the bone surface. Then each angle between the two axial rotations is tested in 0.5 degree increments. At each such angle, the best-fit translation along the axis is found. In this variant, the cost of a harvest location is the sum of the RMS errors for the two surfaces.

\section{Evaluation of the Algorithm}

The algorithm was tested on twelve in-vivo sheep knees for which data was gathered as part of a larger study. Each knee was scanned using a CT arthrogram with a slice thickness of $0.625 \mathrm{~mm}$. The bone and cartilage surfaces were manually segmented from the CT images. In a minimally invasive surgery, a cartilage defect was induced on the medial condyle with a calibrated impact. Three months later, a second CT arthrogram was taken and the bone and cartilage surfaces manually segmented.

The post-defect models were used for planning and the resulting plans were evaluated in comparison to the pre-defect models. Three conditions were tested for each knee: 
- An expert human operator, who had substantial experience planning mosaic arthroplasty, manually produced a surgical plan. The operator matched only the cartilage surface and did not attempt to match the bone surface.

- An inexperienced operator used the planning algorithm to produce a surgical plan that optimized the fit of only the cartilage surface.

- The inexperienced operator used the planning algorithm to produce a surgical plan that optimized the fit of both the cartilage surface and the bone surface.

Each plan was evaluated for surface congruency by computing the RMS error between the planned cartilage surface and the pre-defect cartilage surface of the pre-defect CT arthrogram. The RMS calculation used six points on the circumference and one point at the center of each plug surface. A similar RMS calculation was made for congruency at the bone surface. The percentage of the defect surface covered by plugs was calculated. The total planning time was recorded.

\section{$5 \quad$ Results}

Table 1 summarizes the results. For manual and automatic planning that considered only the cartilage surface (evaluating significance with one-sided t-tests):

- No significant difference was found in the RMS errors (manual $0.31 \mathrm{~mm}$, automatic $0.25 \mathrm{~mm}, \mathrm{p}=0.085$ ), although the low $\mathrm{p}$ value is suggestive of a trend in favor of automatic planning.

- Automatic planning shows a tighter variance in RMS error $( \pm 0.06$, min 0.13 , max 0.38$)$ than manual planning $( \pm 0.10$, min 0.09 , max 0.61$)$, but not to a statistically significant degree using Levene's test for equal variances ( $\mathrm{p}$ $=0.17$ ).

- No significant difference was found in the coverage of the defect surface (manual $84 \%$, automatic $88 \%, \mathrm{p}=0.10$ ), although the trend is also in favor of automatic planning.

- There was significantly less plug overlap with automatic planning (manual $16.1 \%$, automatic $9.7 \%, \mathrm{p}=0.02)$.

- The automatic planning time was substantially faster (4.5 minutes) than the reported manual planning time (30 to 45 minutes), but we cannot make any statistical claims because the manual planning time was not measured accurately.

When the automatic planner optimized both the cartilage and bone surfaces, the defect coverage remained the same at $88 \%$ and the total planning time remained the same at 4.5 minutes. The RMS error of the cartilage surface increased, as one would expect when the bone surface also had to be considered in the optimization. Interestingly, the overall (bone plus cartilage) RMS error was less when only cartilage was considered (although not by a statistically significant amount, so we cannot attribute any meaning to this). 
Table 1. Results showing, for each measure, "average $\pm 95 \%$ confidence interval". In the Condition column, " $\mathrm{C}$ " denotes cartilage only and "C+B" denotes cartilage and bone. * Unfortunately, the manual planning was not timed and the 30 to 45 minutes shown in the table is an estimate provided by the operator. Automatic planning time includes the time for the operator to set up the spline and regions.

\begin{tabular}{l|c|c|c|c|c} 
Condition & $\begin{array}{c}\text { RMSE cartilage } \\
(\mathrm{mm})\end{array}$ & $\begin{array}{c}\text { RMSE cartilage } \\
\text { and bone }(\mathrm{mm})\end{array}$ & $\begin{array}{c}\text { Coverage } \\
(\%)\end{array}$ & $\begin{array}{c}\text { Overlap } \\
(\%)\end{array}$ & $\begin{array}{c}\text { Time } \\
(\text { minutes })\end{array}$ \\
\hline Manual, C & $0.31 \pm 0.10$ & $0.31 \pm 0.07$ & $84 \pm 7$ & $16.1 \pm 4.9$ & $30-45^{*}$ \\
Auto, C & $0.26 \pm 0.06$ & $0.27 \pm 0.07$ & $88 \pm 3$ & $9.7 \pm 4.2$ & $4.5 \pm 1.7$ \\
Auto, C+B & $0.35 \pm 0.09$ & $0.29 \pm 0.06$ & $88 \pm 3$ & $9.7 \pm 4.2$ & $4.5 \pm 1.9$
\end{tabular}

\section{Conclusions}

We have described a computer algorithm to automatically develop a surgical plan for computer assisted mosaic arthroplasty. No statistically significant difference was found between the RMS error of automatic planning and manual planning, although the trends suggest that automatic planning gives better coverage, is more accurate, and has less variance. Automatic planning produces less plug overlap (a good feature) and the algorithm takes much less time than a highly trained human expert and can be used by an inexperienced operator. These results suggest that the automatic planner should be used in place of manual planning, with the plans being subject to final approval from the surgeon.

In ongoing work, we are augmenting the automatic planner with multiple plug radii and non-perpendicular plugs at the defect site, and will shortly be using the automatic planner in human surgeries.

Acknowledgements. Thank you to the reviewers for their many helpful comments. This work is supported by Natural Sciences and Engineering Research Council of Canada (NSERC) grant STPGP 336797 and by the joint NSERC/ Canadian Institutes of Health Research CHRP grant 385959.

\section{References}

1. Halser, E., Herzog, W., Wu, J.Z., Müller, W., Wyss, U.: Articular cartilage biomechanics: theoretical models, material properties, and biosynthetic response. Crit. Rev. Biomed. Eng. 27(6), 415 (1999)

2. Yamashita, F.: The transplantation of an autogeneic osteochondral fragment for osteochondritis dissecans of the knee. Clin. Orthop. Relat. Res. (201), 43 (1985)

3. Hangody, L., Kish, G., Krpti, Z., Szerb, I., Udvarhelyi, I.: Arthroscopic autogenous osteochondral mosaicplasty for the treatment of femoral condylar articular defects: A preliminary report. Knee Surgery, Sports Traumatology, Arthroscopy 5(4), 262-267

4. Pearce, S., Hurtig, M., Clarnette, R., Kalra, M., Cowan, B., Miniaci, A.: An investigation of 2 techniques for optimizing joint surface congruency using multiple cylindrical osteochondral autografts. Arthroscopy: The Journal of Arthroscopic and Related Surgery 17(1), 50-55 (2001) 
5. Koh, J., Kowalski, A., Lautenschlager, E.: The effect of angled osteochondral grafting on contact pressure: A biomechanical study. American Journal of Sports Medicine 34(1), 116-119 (2006)

6. Huang, F., Simonian, P., Norman, A., Clark, J.: Effects of small incongruities in a sheep model of osteochondral autografting. American Journal of Sports Medicine 32(8), 1842-1848 (2004)

7. Kunz, M., Hurtig, M., Waldman, S., Devlin, S., Rudan, J., Bardana, D., Stewart, J.: Image-guided surgical techniques for cartilage repair - an animal trial. In: World Congress of the International Cartilage Repair Society (ICRS), p. 190 (2010)

8. Radermacher, K., Portheine, F., Anton, M., Zimolong, A., Kaspers, G., Rau, G., Staudte, H.W.: Computer assisted orthopaedic surgery with image based individual templates. Clin. Orthop. Relat. Res. (354), 28-38 (1998)

9. Kunz, M., Devlin, S., Rudan, J., Waldman, S., Stewart, J., Hurtig, M.: Computerassisted planning for mosaic arthroplasty. Computer Assisted Radiology and Surgery, S102-S103 (2009)

10. Jakob, R., Franz, T., Gautier, E., Mainil-Varlet, P.: Autologous osteochondral grafting in the knee: indication, results, and reflections. Clinical Orthopaedics and Related Research 401, 170-184 (2002)

11. Koulalis, D., Benedetto, P.D., Citak, M., Loughlin, P., Pearle, A., Kendoff, D.: Comparative study of navigated versus freehand osteochondral graft transplantation of the knee. American Journal of Sports Medicine 37(4), 803-807 (2009)

12. Bale, R., Hoser, C., Rosenberger, R., Rieger, M., Benedetto, K., Fink, C.: Osteochondral lesions of the talus: Computer-assisted retrograde drilling - feasibility and accuracy in initial experiences. Radiology 218, 278 (2001)

13. Hoser, C., Bichler, O., Bale, R., Rosenberger, R., Rieger, M., Kovacs, P., Lang, T., Fink, C.: A computer assisted surgical technique for retrograde autologous osteochondral grafting in talar osteochondritis dissecans (ocd): a cadaveric study. Knee Surgery, Sports Traumatology, Arthroscopy 12(1), 65-71 (2004)

14. Marymount, J., Shute, G., Zhu, H., Varner, K., Paravic, V., Haddad, J., Noble, P.: Computerized matching of autologous femoral grafts for the treatment of medial talar osteochondral defects. Foot and Ankle Intl. 26(9), 708 (2005)

15. Bartz, R., Kamaric, E., Noble, P., Lintner, D., Bocell, J.: Topographic matching of selected donor and recipient sites for osteochondral autografting of the articular surface of the femoral condyles. American Journal of Sports Medicine 29(2), 207$212(2001)$

16. Böttcher, P., Zeissler, M., Grevel, V., Oechtering, G.: Computer simulation of the distal aspect of the femur for assessment of donor core size and surface curvature for autologous osteochondral transplantation in the canine stifle joint. Veterinary Surgery 39(3), 371-379 (2010)

17. Kunz, M., Devlin, S., Gong, R.H., Inoue, J., Waldman, S.D., Hurtig, M., Abolmaesumi, P., Stewart, J.: Prediction of the repair surface over cartilage defects: A comparison of three methods in a sheep model. In: Yang, G.-Z., Hawkes, D., Rueckert, D., Noble, A., Taylor, C. (eds.) MICCAI 2009. LNCS, vol. 5761, pp. 75-82. Springer, Heidelberg (2009)

18. Haklar, U., Tuzuner, T., Uygur, I., Kocaoglu, B., Guven, O.: The effect of overlapping on the primary stability of osteochondral grafts in mosaicplasty. Knee Surgery, Sports Traumatology, Arthroscopy 16(7), 651-654 (2008) 